\title{
Communication
}

\section{Intraosseous Squamous Cell Carcinoma Associated with Denosumab-Induced Osteonecrosis of the Jaw}

\author{
Sandra Bacián Martínez ${ }^{1}$, Carlos Navarro Cuéllar ${ }^{1, * \mathbb{C}}$, Carlos Navarro Vila ${ }^{1}$, Edurne Palacios Weiss ${ }^{1}$, \\ Roser Pujol Romanyá ${ }^{1}$, Álvaro del Amo Fernández de Velasco ${ }^{1}$, Margarita Escrig de Teijeiro ${ }^{1}$, \\ José Luis del Castillo Pardo de Vera ${ }^{1}$, Irene Rodríguez Pérez ${ }^{2}$, Arturo Sánchez-Pérez ${ }^{3}{ }^{\mathbb{D}}$, José J. Zamorano-León ${ }^{4}$, \\ Antonio López-Farré ${ }^{4}$, María Isabel García-Hidalgo Alonso ${ }^{5}$ and Ignacio Navarro Cuéllar ${ }^{1}$
}

check for updates

Citation: Bacián Martínez, S.; Navarro Cuéllar, C.; Navarro Vila, C.; Palacios Weiss, E.; Pujol Romanyá, R.; del Amo Fernández de Velasco, Á.; Escrig de Teijeiro, M.; del Castillo Pardo de Vera, J.L.;

Rodríguez Pérez, I.;

Sánchez-Pérez, A.; et al. Intraosseous Squamous Cell Carcinoma Associated with Denosumab-Induced Osteonecrosis of the Jaw. Appl. Sci. 2021, 11, 7646. https://doi.org/ 10.3390/app11167646

Academic Editor: Gabriele Cervino

Received: 30 June 2021

Accepted: 18 August 2021

Published: 20 August 2021

Publisher's Note: MDPI stays neutral with regard to jurisdictional claims in published maps and institutional affiliations.

Copyright: (c) 2021 by the authors. Licensee MDPI, Basel, Switzerland. This article is an open access article distributed under the terms and conditions of the Creative Commons Attribution (CC BY) license (https:/ / creativecommons.org/licenses/by/ $4.0 /)$.
1 Maxillofacial Surgery Department, Hospital Universitario HLA Moncloa, 28008 Madrid, Spain; sbacian@gmail.com (S.B.M.); hazamachado2@gmail.com (C.N.V.); edurne.palacios@gmail.com (E.P.W.); roserpujol@gmail.com (R.P.R.); alvaro_delamo@yahoo.es (Á.d.A.F.d.V.); margaritaescrig@gmail.com (M.E.d.T.); delcastillo6@hotmail.com (J.L.d.C.P.d.V.); nnavcu@hotmail.com (I.N.C.)

2 Pathology Department, Hospital Universitario HLA Moncloa, 28008 Madrid, Spain; irodriguez@analizalab.com

3 Faculty of Medicine, Murcia University, 30008 Murcia, Spain; arturosa@um.es

4 Faculty of Medicine, Universidad Complutense de Madrid, 28040 Madrid, Spain; josej.zamorano@gmail.com (J.J.Z.-L.); ajlf@telefonica.net (A.L.-F.)

5 Radiology Department, Hospital Puerta de Hierro, 28222 Madrid, Spain; mabelgha@gmail.com

* Correspondence: cnavarrocuellar@gmail.com

Abstract: Medication-related osteonecrosis of the jaw (MRONJ) has been associated with the use of different drugs administered in the treatment of malignant neoplasms or metastases and in antiresorptive therapies. Since 2010, denosumab, a monoclonal antibody whose mechanism of action is to prevent the activation of the RANK receptor on the surface of osteoclasts, has been associated with osteonecrosis of the jaw. Primary intraosseous squamous cell carcinoma (PIOSCC) is a rare neoplasm characterised by the presence of squamous cells within the maxillary or mandibular bone without an initial clinical association with the oral mucosa. We present a case of a PIOSCC in an osteonecrosis of the jaw related to a patient who received antiresorptive treatment with denosumab outlining the clinical and histopathological features of MRONJ and PIOSCC.

Keywords: denosumab; medication related osteonecrosis of the jaw (MRNOJ); primary intraosseous squamous cell carcinoma

\section{Introduction}

Head and neck carcinomas represent $5-7 \%$ of all cancers in the world, with epithelial neoplasms accounting for about $90 \%$ of cases. Squamous cell carcinoma is a cancer of the oral cavity and oropharynx and constitutes around $3 \%$ of all malignant tumours in the body [1].

Primary intraosseous squamous cell carcinoma (PIOSCC) is a rare neoplasm characterized by the presence of squamous cells within the maxillary or mandibular bone without an initial clinical association with the oral mucosa [2]. It was initially described by Loos in 1913, later receiving different nomenclatures such as intraalveolar epidermoid carcinoma or primary intraalveolar epidermoid carcinoma [3]. From a histological point of view, PIOSCC presumably originates from dental epithelial debris and can therefore be associated with the development of odontogenic cysts or odontogenic tumours [2].

Medication-related osteonecrosis of the jaw (MRONJ) was first reported by Marx in 2003, who described the association of this pathology with the intake of bisphosphonates. [4]. Since then, MRNOJ has been related to the use of different drugs administered in the treatment of malignant neoplasms or metastases and in antiresorptive therapies [5]. 
Recently, the association of this pathology with other drugs such as simvastatin has been reported [6].

In 2010, cases of osteonecrosis of the jaw associated with denosumab, a monoclonal antibody whose mechanism of action is to prevent the activation of the RANK receptor on the surface of osteoclasts, began to be published in the scientific literature [7].

The aim of this article is to present a case of a patient who developed a PIOSCC in an osteonecrotic mandibular lesion after being diagnosed of denosumab-related osteonecrosis of the jaw. For this purpose, the clinical and histopathological features of MRONJ and PIOSCC are described as well as our therapeutic management according to the clinical classification defined by the American Association of Oral and Maxillofacial Surgeons (AAOMS) [8].

To our knowledge, this is the first time that this malignant entity has been associated with denosumab-related osteonecrosis of the jaw. The study was endorsed by the Hospital Ethics Committee at Hospital Universitario HLA Moncloa, Madrid, Spain (protocol code maxilohm 11/2020).

\section{Case Presentation}

The patient was a 70-year-old woman suffering from type II diabetes mellitus, arterial hypertension, dyslipidaemia, vitamin B12 deficiency, and osteoporosis. The patient had been treated with denosumab, at a dose of $60 \mathrm{mg}$ every 6 months for more than 2 years and was referred to the Oral and Maxillofacial Surgery Department for evaluation of suspected mandibular osteonecrosis after multiple tooth extractions that had been performed at another centre.

Physical examination revealed a pathological mandibular fracture with an extensive area of intraoral bone exposure. External examination revealed a cervical skin fistula (Figure 1).

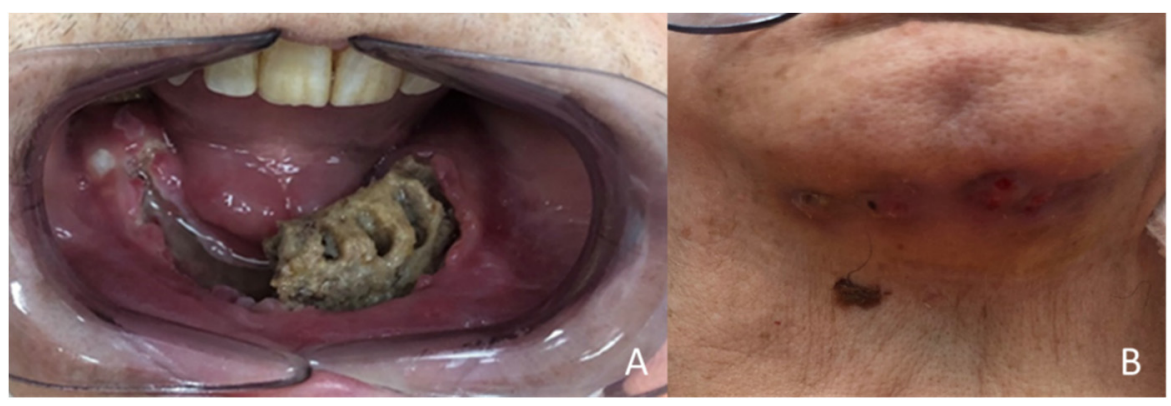

Figure 1. (A) Mandibular osteonecrosis. Intraoral view. (B) Extraoral cutaneous submental fistula.

Following the usual protocol with these patients, it was decided to randomly perform superficial and deep intraoral bone biopsies as well as intraoral (mucosa) and extraoral (skin) perilesional soft tissue biopsies. Imaging tests were requested. The anatomopathological result was bone necrosis and inflammatory granulation tissue (Figure 2).

Orthopantomography and computed tomography (CT) showed (Figure 3) a diffuse lytic involvement which was compatible with mandibular necrosis that extended from the left to the right mandibular angles, and the presence of the pathological fracture described above. No pathological lymphatic neck nodes were described, and no distant lesions were diagnosed in a whole-body CT scan. Due to negative bone and soft tissue biopsies (a diagnosis of carcinoma could not be established) and with the CT scan findings suggesting osteonecrosis of the jaw, it was not considered necessary to request an MRI scan. This decision was made in agreement with the Neuroradiology Department. 


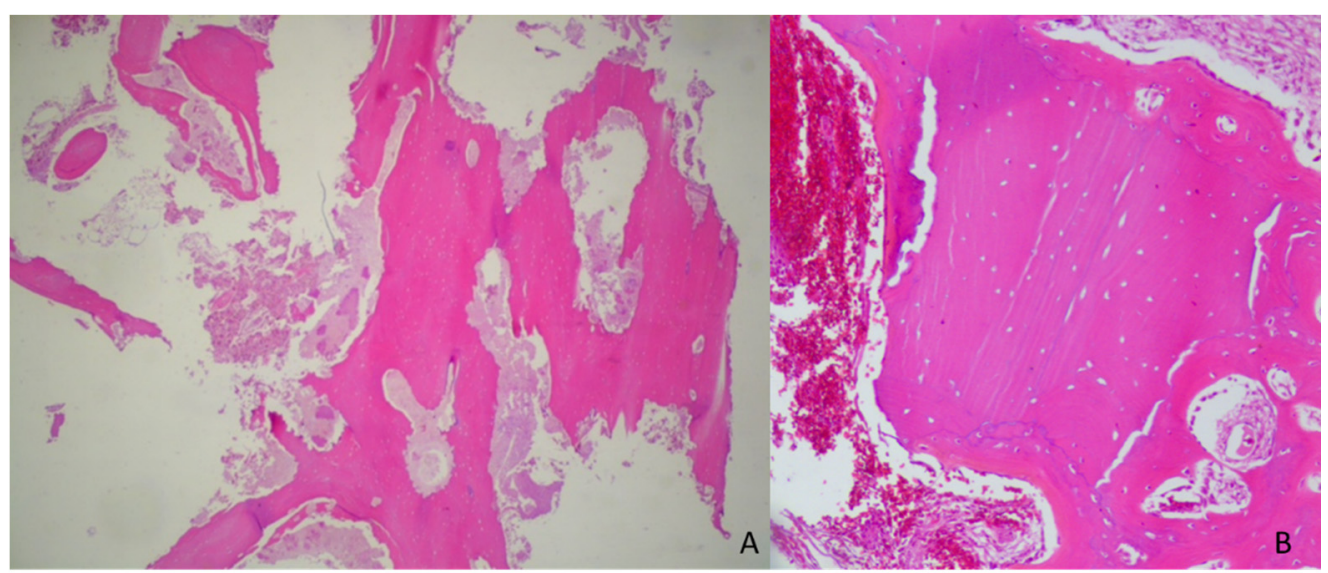

Figure 2. (A) Hematoxylyn-eosin-stained section showing necrotic bony trabeculae demonstrating empty osteocyte lacunae necrotic devoid of inflammatory cells (original magnification $\times 2.5$ ). (B) A border of new bone formation is identified with a superficial reactive line where osteoblasts can be seen (stain, H\&E; magnification, $\times 10$ ).

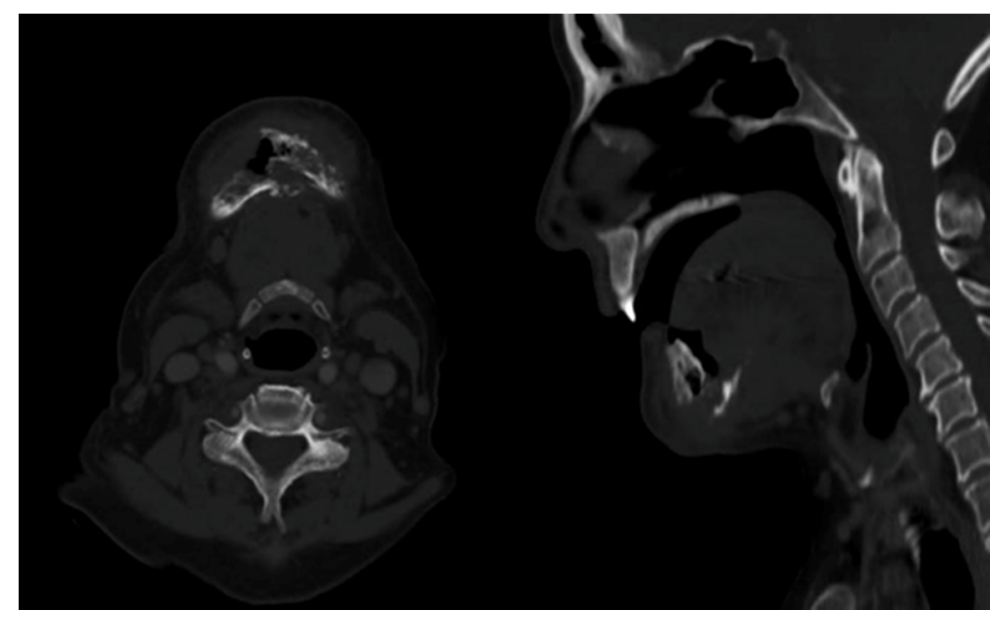

Figure 3. Axial and sagittal CT scan demonstrating mandibular necrosis and pathologic fracture.

With the suspected diagnosis of drug-associated osteonecrosis of the jaw, further studies were performed to evaluate microsurgical bone reconstruction with a fibula free flap. AngioCT of supra-aortic and tibioperoneal vessels ruled out pathology or anatomical variants in both vascular axes without clearly identifying perforating vessels from the vascular pedicle to the skin of the legs.

The jaw resection surgery and the reconstruction with the fibula flap were virtually planned in order to design bone cutting guides in the mandibular area and in the lower limb (Figure 4).

Based on this information, a custom-made titanium implant was designed to fix the free flap to the remanent mandible (Figure 5). 

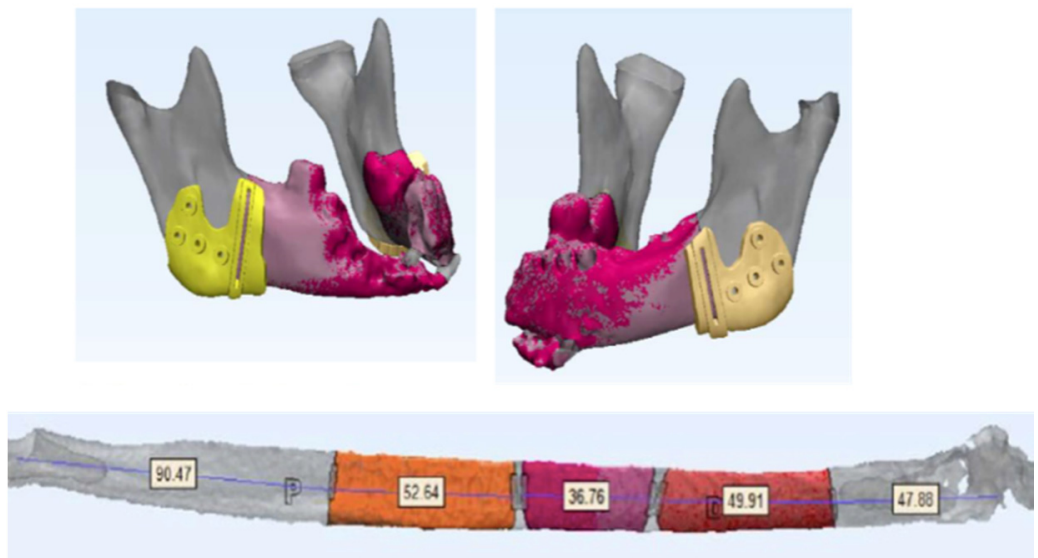

Figure 4. Virtual surgical planning. Surgical cutting guides and design of fibula osteotomies for mandibular reconstruction.
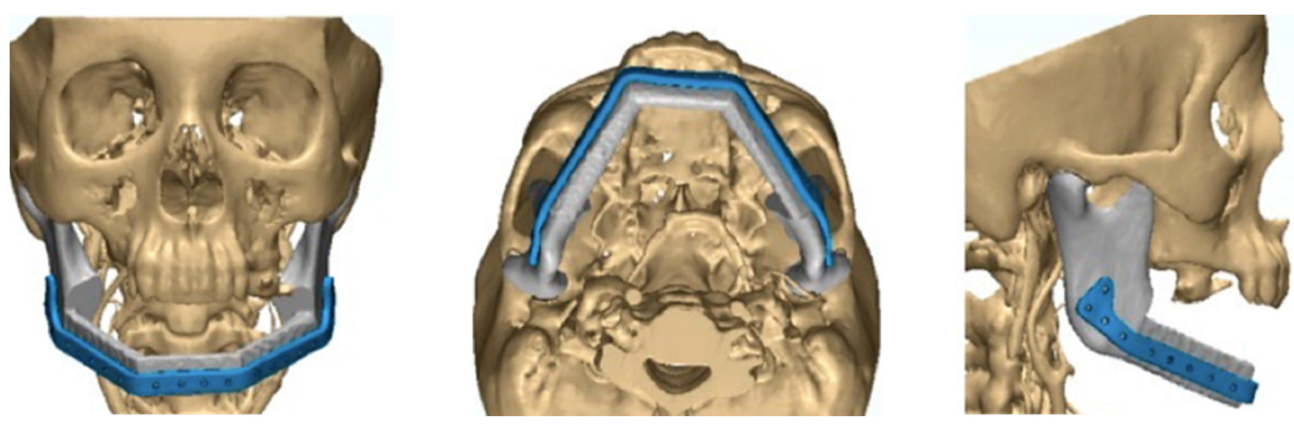

Figure 5. Customized titanium 2.0 plate design.

The patient underwent surgery by means of a tracheotomy, an external cervical approach, placement of the cutting guides, segmental angle-to-angle mandibulectomy, and immediate reconstruction with a fibula free flap (Figure 6).
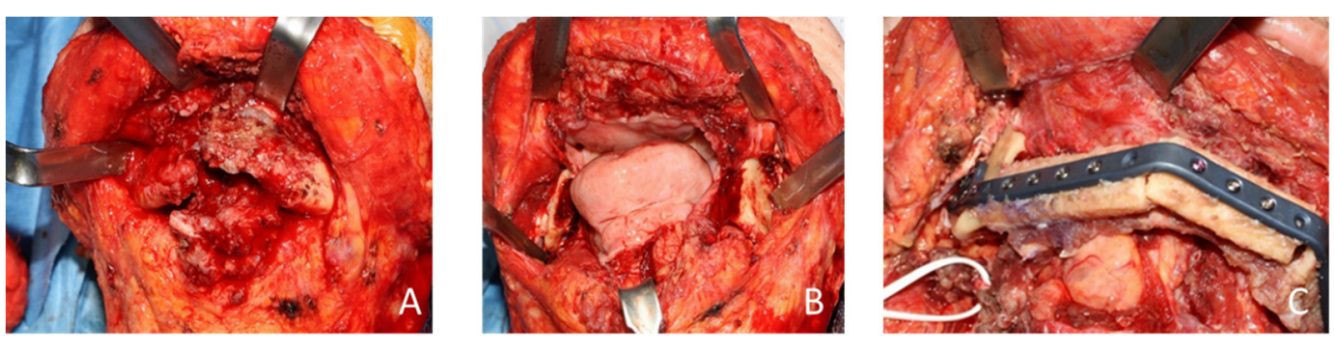

Figure 6. (A) Intraoperative image of jaw osteonecrosis. (B) Segmental mandibulectomy before reconstruction. (C) Fibula flap reconstruction with customized titanium implant used for internal fixation.

The recipient vessels for the microsurgical anastomoses were the external carotid artery and two veins (the thyrolinguofacial trunk and the external jugular vein). Internal fixation of the custom-made titanium implant was performed with 2.0 screws. Intraoral soft tissue closure was achieved with two buccinator myomucosal flaps.

The final histopathological specimen revealed a highly mature intraosseous squamous cell carcinoma surrounded by necrotic tissue with single focal cortical bone involvement. This epithelial neoplasm was organized in solid nests or cords with medium-sized cells with large eosinophilic cytoplasm and well-defined membrane, large and vesicular nuclei, and the presence of nucleolus. Keratin formation and some corneal pearls were observed. The bone margins were free of disease. A primary intraosseous squamous cell carcinoma 
was diagnosed (Figure 7). It was decided not to give adjuvant treatment, as clear margins were encountered, and the involvement was focal. The patient developed regional and systemic progression of the disease at 9 months, underwernt chemotherapy treatment, and deceased 21 months after the initial diagnosis.

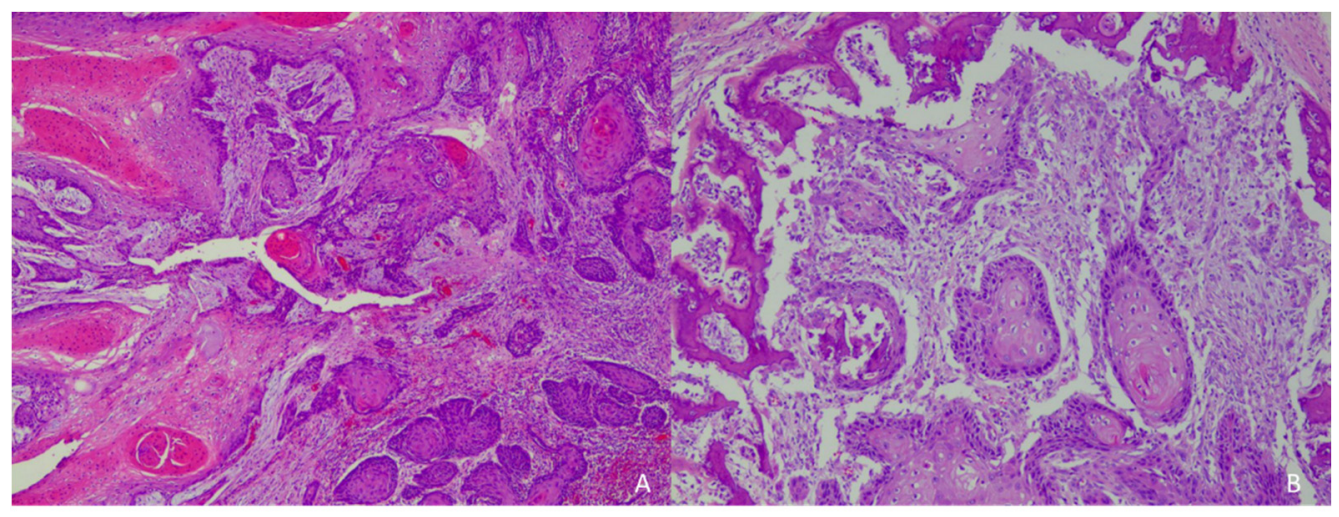

Figure 7. (A) Hematoxylyn-eosin-stained section from the lesion showing invasive squamous cell carcinoma (SCC) characterized by sheets and nest of squamous epithelial cells containing abundant eosinophilic cytoplasm, round to oval nuclei, and prominent nucleoli. They show keratinization and horn pearl formation (original magnification $\times 10$ ). (B) SCC bone invasion site (stain, H\&E; magnification, $\times 10)$.

\section{Discussion}

PIOSCC is a rare neoplasm characterized by the presence of intraosseous squamous cells that are probably derived from residual odontogenic epithelium [2]. The World Health Organisation (WHO) has divided PIOSCC into three types: solid-type carcinoma, carcinoma arising from a keratocystic odontogenic tumour, and carcinoma arising from an odontogenic cyst [3].

The differential diagnosis of PIOSCC should include carcinomas of an odontogenic nature, such as malignant ameloblastoma, SCC of the overlying oral mucosa, and metastases of other primary neoplasms in the jaw. It should be taken in consideration that in up to $5 \%$ of patients with mandibular malignant cells, these are not detectable on imaging tests [9] and that whenever a diagnosis of PIOSCC is given, the existence of a distant primary tumour should be excluded [2]. The patient described in this article had a whole-body negative tumour CT scan.

The term MRONJ was introduced by the American Association of Oral and Maxillofacial Surgeons (AAOMS 2014) to include all forms of drug-induced osteonecrosis of the jaw [8].

The following criteria must be addressed for a clinical diagnosis of MRONJ:

1. Presence of exposed bone (or bone that can be probed through an intraoral or extraoral fistula) in the maxillofacial region for a period of 8 weeks.

2. Current or previous treatment with antiresorptive agents (bisphosphonates or denosumab) or antiangiogenic agents.

3. No history of radiotherapy or metastatic disease in the jaw.

Although histological criteria are not included in this definition, routine histological confirmation should be performed to support the diagnosis and to exclude malignancy $[9,10]$.

The risk of developing osteonecrosis of the jaw is $0.01 \%$ in patients with osteoporosis and up to $15 \%$ in patients who have been treated with high doses of bisphosphonates or antiresorptives for malignant diseases $[10,11]$. Risk factors associated with its development are oral surgery procedures, odontogenic infections, and concurrent treatment with chemotherapeutic agents or corticosteroids. 
Denosumab is a drug that is included in the category of monoclonal antibodies (IgG2) and is used in pathologies with a high risk of bone fractures such as osteoporosis in postmenopausal women and bone loss associated with hormonal suppression in men with prostate cancer [11]. Its mechanism of action is due to its binding to the receptor activator of nuclear factor kappa-B ligand (RANK-L), preventing the maturation, function, and survival of osteoclasts, thus improving bone mineral density and reducing the probability of fracture [12]. This prevents the interaction of RANK-L/RANK and inhibits the formation, function, and survival of osteoclasts, causing a decrease in bone resorption in trabecular and cortical bone.

The risk of developing MRONJ is higher in patients who require a higher dose of administration and an intake period longer than 2 years [13]. Although the literature reports a low incidence of MRONJ resulting from the use of denosumab, when it does occur, it involves severe functional and masticatory disorders, with a major impact on the patient's quality of life. It is noteworthy that most the adverse effects of denosumab occur at doses of $120 \mathrm{mg}$, while side effects at doses of $60 \mathrm{mg}$ are directly related to the duration of treatment [12].

Stopeck et al. [14] report that monthly subcutaneous injections of denosumab at a dosage of $120 \mathrm{mg}$ were more effective than zoledronic acid ( $4 \mathrm{mg}$ monthly) in patients with breast cancer and bone metastases. The authors describe few cases of MRONJ, with most of them having previous risk factors such as tooth extraction or poor oral hygiene. These findings are consistent with the work of Henry et al. [15], in which the incidence of MRONJ was low, similar in both treatment groups (denosumab and zolendronic acid), and mostly associated with risk factors.

In a patient with suspected MRONJ, an adequate medical report with information about the treatments that have been administered as well as their duration should be recorded. The appearance and progression of bone lesions (clinical or radiology) or soft tissue lesions should orient the diagnosis towards a malignant pathology. Biopsy is mandatory in clinical cases suspected of MRNOJ in order to rule out malignancy, and it is recommended to include perilesional soft tissues, affected bone, and healthy bone. The presented case was initially managed with several soft tissue and bone biopsies without obtaining a diagnosis of malignancy.

In a case of PIOSCC or MRNOJ, imaging tests (orthopantomography, CT, MRI) should complete the diagnosis. The described patient was examined using orthopantomography, a head and neck CT scan, a body CT scan, and an angio-CT scan of the supra-aortic and tibio-peroneal vessels.

MRNOJ treatment will depend on the symptomatology and extent of necrosis, whereas the treatment of PIOSCC, as long as there is no evidence of distant disease, will require excision with safety margins, lymphadenectomy depending on the stage of disease, and primary reconstruction, if possible. This patient was treated with a segmental angle to angle mandibulectomy and primary reconstruction with a free fibula flap.

To our knowledge, this is the first case report of a mandibular primary intraosseous squamous cell carcinoma diagnosed in the context of a denosumab-associated mandibular necrosis.

\section{Conclusions}

Initial management of MRONJ should always be performed with biopsies prior to any further treatment. Mandibular PIOSCC is a rare clinical entity that can occur de novo in the context of MRONJ. Treatment will depend on the extent of the disease, both loco-regionally and systemically. 
Author Contributions: Conceptualization, S.B.M. and C.N.C.; methodology, C.N.V.; software, E.P.W., J.L.d.C.P.d.V.; validation, S.B.M., C.N.C., I.N.C. and A.S.-P.; investigation, R.P.R., M.E.d.T. and I.R.P.; resources, I.R.P.; data curation, Á.d.A.F.d.V.; writing-original draft preparation, S.B.M.; writing-review and editing, S.B.M. and I.N.C.; visualization, C.N.C.; supervision, C.N.C.; project administration, C.N.C.; supervision and visualization, M.I.G.-H.A.; methodology and visualization, J.J.Z.-L.; supervision and visualization, A.L.-F. All authors have read and agreed to the published version of the manuscript.

Funding: This research received no external funding.

Institutional Review Board Statement: The study was conducted according to the guidelines of the Declaration of Helsinki and was endorsed by the Hospital Ethics Committee at Hospital Universitario HLA Moncloa, Madrid, Spain (protocol code maxilohm 11/2020).

Informed Consent Statement: Written informed consent has been obtained from the patient to publish this paper.

Conflicts of Interest: The authors declare no conflict of interest.

\section{References}

1. Sung, H.; Ferlay, J.; Siegel, R.L.; Laversanne, M.; Soerjomataram, I.; Jemal, A.; Bray, F. Global Cancer Statistics 2020: GLOBOCAN Estimates of Incidence and Mortality Worldwide for 36 Cancers in 185 Countries. CA Cancer J. Clin. 2021, 71, 209-249. [CrossRef] [PubMed]

2. Woolgar, J.A.; Triantafyllou, A.; Ferlito, A.; Devaney, K.O.; Lewis, J.S.; Rinaldo, A.; Slootweg, P.J.; Barnes, L. Intraosseous carcinoma of the jaws: A clinicopathologic review. Part III: Primary intraosseous squamous cell carcinoma. Head Neck 2013, 35, 906-909. [CrossRef] [PubMed]

3. Li, K.; Yang, L.; Qiao, Y.-J.; Liang, Y.-J.; Wang, X.; Liao, G.-Q. Risk factors and prognosis for the primary intraosseous carcinoma of the jaw. Int. J. Oral Maxillofac. Surg. 2019, 48, 157-162. [CrossRef] [PubMed]

4. Marx, R. Pamidronate (Aredia) and zolendronate (Zometa) induced avascular necrosis of the jaws: A growing epidemic. J. Oral Maxilofac. Surg. 2003, 61, 1115-1118. [CrossRef]

5. Tocaciu, S.; Breik, O.; Lim, B.; Angel, C.; Rutherford, N. Diagnostic dilemma between medication-related osteonecrosis and oral squamous cell carcinoma in a mandibular lytic lesion. Br. J. Oral Maxillofac. Surg. 2017, 55, e53-e57. [CrossRef] [PubMed]

6. Samieirad, S.; Labafchi, A.; Famili, K.; Hashemzadeh, H. Medication-Related Osteonecrosis of the Jaw (MRONJ) due to Simvastatin: An Unusual Case Report. World J. Plast. Surg. 2021, 10, 132-135. [PubMed]

7. Qaisi, M.; Hargett, J.; Loeb, M.; Brown, J.; Caloss, R. Denosumab Related Osteonecrosis of the Jaw with Spontaneous Necrosis of the Soft Palate: Report of a Life Threatening Case. Case Rep. Dent. 2016, 2016, 5070187. [CrossRef] [PubMed]

8. Ruggiero, S.L.; Dodson, T.B.; Fantasia, J.; Goodday, R.; Aghaloo, T.; Mehrotra, B.; O’Ryan, F. American association of oral and maxillofacial surgeons position paper on medication-related osteonecrosis of the jaw-2014 update. J. Oral Maxillofac. Surg. 2014, 72, 1938-1956. [CrossRef] [PubMed]

9. Owosho, A.A.; Xu, B.; Kadempour, A.; Yom, S.K.; Joseph Randazzo, J.; Ghossein, R.A.; Huryn, J.M.; Estilo, C.L. Metastatic solid tumors to the jaw and oral soft tissue: A retrospective clinical analysis of 44 patients from a single institution. J. Craniomaxillofac. Surg. 2016, 44, 1047-1053. [CrossRef] [PubMed]

10. Krestan, C.R.; Mitchell, D.A.; Kirschke, J.S.; Wutzl, A.; Mücke, T. Bisphosphonate and Medication-Related Osteonecrosis of the Jaw: A Review. Semin. Musculoskelet. Radiol. 2016, 20, 305-314. [CrossRef] [PubMed]

11. Diaz-Reverand, S.A.; Naval-Gíaz, L.; Muñoz-Guerra, M.F.; Sastre-Pérez, J.; Rodríguez-Campo, F.J.; Gil-Diez, J.L. Manejo de la osteonecrosis maxilar asociada al uso de medicamentos en virtud de su estadio clínico: Análisis de 19 casos. Rev. Española Cirugía Oral Maxilofac. 2018, 40, 104-111. [CrossRef]

12. Boquete-Castro, A.; Gómez-Moreno, G.; Calvo-Guirado, J.L.; Aguilar-Salvatierra, A.; Delgado-Ruiz, R.A. Denosumab and osteonecrosis of the jaw. A systematic analysis of events reported in clinical trials. Clin. Oral Implant. Res. 2016, 27, 367-375. [CrossRef] [PubMed]

13. Saad, F.; Brown, J.E.; Van Poznaketal, C. Incidence, risk factors, and outcomes of osteonecrosis of the jaw: Integrated analysis from three blinded active-controlled phase III trials in cancer patients with bone metastases. Ann. Oncol. 2012, 23, 1341-1347. [CrossRef] [PubMed]

14. Stopeck, A.T.; Lipton, A.; Body, J.-J.; Steger, G.G.; Tonkin, K.; De Boer, R.H.; Lichinitser, M.; Fujiwara, Y.; Yardley, D.A.; Viniegra, M.; et al. Denosumab Compared With Zoledronic Acid for the Treatment of Bone Metastases in Patients With Advanced Breast Cancer: A Randomized, Double-Blind Study. J. Clin. Oncol. 2010, 28, 5132-5139. [CrossRef] [PubMed]

15. Henry, D.; Vadhan-Raj, S.; Hirsh, V.; Von Moos, R.; Hungria, V.; Costa, L.; Woll, P.; Scagliotti, G.; Smith, G.; Feng, A.; et al. Delaying skeletal-related events in a randomized phase 3 study of denosumab versus zoledronic acid in patients with advanced cancer: An analysis of data from patients with solid tumors. Support. Care Cancer 2014, 22, 679-687. [CrossRef] [PubMed] 